Original Article - Clinical Science

\title{
Predictors of visual outcome and the role of early vitrectomy in Streptococcal endophthalmitis
}

\author{
Emil D Kurniawan MBBS, ${ }^{1}$ John R Rocke MBBS, ${ }^{1}$ Sukhpal S Sandhu MD FRANZCO ${ }^{1,2}$ \\ and Penelope $\mathrm{J}$ Allen MBBS FRANZCO ${ }^{1,2}$
}

${ }^{1}$ The Royal Victorian Eye and Ear Hospital, 32 Gisborne Street, East Melbourne, VIC, Australia

${ }^{2}$ Centre for Eye Research Australia, University of Melbourne, Level 7, 32 Gisborne Street, East Melbourne, VIC, Australia

Correspondence (prior to publication): Dr John Rocke, Unit 2, 98 Smith Street, Collingwood VIC 3066, Australia

Email: john.rocke@hotmail.com

Correspondence (after publication): Dr Penelope J Allen, Royal Victorian Eye and Ear Hospital, 32 Gisborne Street, East Melbourne, Victoria 3002, Australia

Email: pjallen@melbourneretina.com.au

Short running title: Streptococcal endophthalmitis outcomes

Received 21 April 2017; accepted 18 September 2017

Conflict of interest: None

Funding sources: None

This is the author manuscript accepted for publication and has undergone full peer review but has not been through the copyediting, typesetting, pagination and proofreading process, which may lead to differences between this version and the Version of Record. Please cite this article as doi: 10.1111/ceo.13077

This article is protected by copyright. All rights reserved. 
Ethics approval: This study conformed to the tenets of the Declaration of Helsinki, and has been approved by the Ethics Committee of the Royal Victorian Eye and Ear Hospital, Melbourne, Australia. 


\section{ABSTRACT}

Importance: Streptococcus endophthalmitis has devastating sequelae. This study aims to identify factors which may be targeted to optimise patient outcomes.

Background: This study investigated characteristics influencing visual outcomes, and the role of early vitrectomy.

Design: Retrospective observational case series of consecutive patients Participants: All patients with a culture-positive diagnosis of Streptococcal endophthalmitis treated at a tertiary ophthalmology referral centre between July 1997 and February 2012

Method: Patient records were reviewed and data collected on their presentation, examination, microbiology results, procedures and final outcome.

Main outcome measures: Visual acuity (VA) and enucleation/evisceration Results: Of 101 patients, $35.6 \%$ presented with a VA of hand movements (HM) and $42.6 \%$ with light perception (LP). Final VA was poor (6/60 or worse) in $77.6 \%$, and $24.7 \%$ were enucleated/eviscerated. Presenting VA of LP or worse $(p=0.008)$, no view of fundus ( $p=0.001)$, large number of organisms $(p<0.001)$, recognition of Streptococci on gram stain $(p=0.010)$, heavy growth on culture $(p<0.001)$, and more intravitreal injections $(p=0.038)$ were significantly associated with poor visual outcome (6/60 or worse). Presenting VA of LP or worse $(p=0.042)$ and non-viridans Streptococci $(p=0.002)$ were significantly associated with enucleation/evisceration. Fifteen patients $(14.9 \%)$ had early vitrectomy within 48 hours which was not associated with poor final VA or removal of the eye $(p=1.000)$.

Conclusions: Early vitrectomy did not influence visual outcome in this cohort. Microbiology results were useful in predicting poor outcomes, and may allow clinicians to make early treatment decisions and provide prognostic information for patients. 
Keywords: infection, microbiology, treatment surgery, retina 


\section{INTRODUCTION}

Streptococcus species is an important pathogen in endophthalmitis. It is the leading cause of endophthalmitis in late bleb-related endophthalmitis (30-57\%), following intravitreal injections (30.8\%), and is a contributor to post-traumatic endophthalmitis (21.6-26.9\%) ${ }^{1-3}$ It is also the second most common cause of endophthalmitis following cataract surgery (8.2-11.5\%), and is an important pathogen in endogenous endophthalmitis. ${ }^{4,5}$ Streptococcal endophthalmitis often presents with florid inflammation and poor visual acuity, and have worse visual outcomes compared to Staphylococcal endophthalmitis. ${ }^{6-10}$

Currently, most experts advocate intravitreal tap and injection of antibiotics as first line treatment for infective endophthalmitis. While outcomes have improved since intravitreal antibiotics were introduced, there is still controversy regarding other aspects of management such as vitrectomy. Despite this current practise, the role and timing of vitrectomy, the use of systemic antibiotics and intravitreal steroids remain unclear. ${ }^{11-13}$

This study looks at the spectrum of Streptococcal endophthalmitis presenting to a tertiary referral centre over 15 years, from 1997 to 2012. It aims to describe the range of clinical presentation, microbiological results, management and outcomes. The study also looks into factors of presentation, investigation and management that may determine visual outcomes and the likelihood of removal of the eye, in particular the role of early vitrectomy.

\section{METHODS}


Consecutive patients who presented to a single tertiary referral centre, the Royal Victorian Eye and Ear Hospital (RVEEH, Melbourne, Australia) between July 1997 and February 2012 with culture-positive Streptococca/ endophthalmitis were included. The Human Research and Ethics Committee of RVEEH approved the study, and the conduct of the study followed the tenets of the Declaration of Helsinki. Medical records of these patients were reviewed. Examination findings at presentation were gathered, including presenting visual acuity, presence of hypopyon, and whether the fundus was visible on examination. The putative cause was recorded and medical co-morbidities were noted, including diabetes status.

All cases of clinically suspected endophthalmitis underwent a strict protocol of aqueous and vitreous taps, followed by immediate standard intravitreal antibiotic administration (Ceftazidime $2 \mathrm{mg} / 0.1 \mathrm{ml}$, Vancomycin $1 \mathrm{mg} / 0.1 \mathrm{ml}$ ) - known as a "tap and inject". Under sterile technique and local anaesthetic, $0.05-0.10 \mathrm{ml}$ of aqueous humour was aspirated using a 30-gauge needle mounted on a $1 \mathrm{ml}$ syringe. Vitreous tap was preformed using a 25-gauge needle, entering the vitreous cavity at the pars plana ( $4 \mathrm{~mm}$ from the limbus if phakic and $3.5 \mathrm{~mm}$ if pseudophakic), to obtain $0.3 \mathrm{ml}$ of vitreous humour. All ocular samples were sent to a single pathology department in a tertiary centre (St. Vincent's Hospital, East Melbourne).

Microscopy results were noted for gram stain results (positive or negative), amount of organisms (graded as $1+, 2+$ or $3+$ ), morphology (cocci, bacilli or coccobacilli), and whether or not organisms resembled Streptococci (yes or no). Grading for amount of organisms was performed on high-power oil immersion field (x1000) as follows: 0 ( $<1$ per 10 oil immersion fields), $1+$ ( $<1$ per oil immersion field), $2+(1-10$ per oil immersion field), or $3+(>10$ per oil immersion field). 
Culture results were confirmed for presence of Streptococci, and noted for species of Streptococci, amount of growth on culture (light, moderate, heavy), and presence of other microorganisms. The amount of growth on culture plate was graded as follows: none (no growth on culture plate), light (organisms grown in primary inoculum only), moderate (organisms grown in primary and secondary inoculums), or heavy (organisms grown in three or more inoculums).

The management and progress of each case of endophthalmitis were noted in detail. Data was collected on the number and timing of each intravitreal injection, use of intravitreal steroid, vitrectomy and timing of vitrectomy, the use of systemic antibiotics, and if any other procedures (enucleation/evisceration) were performed.

Main outcome measure was the final visual outcome, at least six months after presentation or when vision became stable. The secondary outcome measure was removal of the eye, either by enucleation or evisceration. If follow-up data was not available in patient records, information was obtained through correspondence with treating ophthalmologists.

All statistical analysis was performed on SPSS version 15 (SPSS Inc, Chicago, IL, USA). Categorical data was analysed using the chi-squared test and Fisher's exact test. Where applicable, the Yates' correction was used. Continuous data was stratified into appropriate categorical groups for analysis, unless otherwise noted. Binary logistic regression models were used to analyse the association between patient characteristics and study endpoints.

\section{RESULTS}


Out of 856 patients diagnosed with endophthalmitis in this centre over the 15-year period, $101(11.8 \%)$ were culture-positive for Streptococcus. Most of these cases occurred postoperatively (87.1\%). Cataract surgery accounted for 51 (50.5\%), followed by glaucoma surgery $(24,23.8 \%)$, corneal surgery $(5,5.0 \%)$, intravitreal injection $(6,6.0 \%)$, and vitreoretinal surgery $(2,2.0 \%)$. Among the other causes, seven $(6.9 \%)$ occurred following corneal ulceration, five (5.0\%) following trauma, and one $(1.0 \%)$ was from endogenous spread.

The presenting VA was count fingers (CF) or better in only 10 (9.9\%) of cases. Most patients presented with hand motion (HM) $(36,35.6 \%)$ or light perception (LP) vision (43, 42.6\%), eight (7.9\%) had no light perception (NLP), Table 1. At initial examination, the fundus was not visible in 91 cases (90.1\%). Hypopyon was seen in 76 of 90 patients (84.4\%). 20 patients (19.8\%) had a history of diabetes mellitus.

On microscopy gram stain, type of organisms was reported in 80 cases (79.8\%). The majority were gram-positive cocci, and further identification of gram-positive cocci resembling Streptococci was made in 48 cases (47.5\%). The number of organisms was reported as $1+$ in 12 cases (11.9\%), $2+$ in 18 cases $(17.8 \%)$, and $3+$ in 50 cases (49.5\%). On culture, the most common species was S. pneumoniae (23 cases, 22.8\%); viridans group Streptococci such as S. oralis and S. mitis accounted for 63 cases (62.4\%), which included cases of mixed growth. Growth on culture, regardless of identification of organism, was noted as either light (26 cases, $25.7 \%$ ), moderate (16 cases, $15.8 \%$ ) or heavy (52 cases, $51.5 \%$ ). Antibiotic sensitivity testing was routinely performed. $100 \%$ of strains cultured were susceptible to Vancomycin, $98.7 \%$ to Chloramphenicol, $89.7 \%$ to Cefazolin, $88.1 \%$ to Ofloxacin, $86.5 \%$ to Penicillin, and $4.0 \%$ to Neomycin. 
An initial intravitreal 'tap and inject'was performed in 94 of 101 patients (93.1\%) on the day of admission. Of the seven patients who did not have any intravitreal injections, six proceeded directly to surgery: one had a vitrectomy and lensectomy, four had an evisceration, and one had an enucleation. A single patient received only topical and systemic antibiotics. In total, 15 (14.9\%) patients had a vitrectomy within 48 hours of admission. Overall, $30(29.7 \%)$ patients received one set of intravitreal therapy, $42(41.6 \%)$ patients received two sets, $21(20.8 \%)$ received three sets, and one $(1.0 \%)$ received four. Intravitreal dexamethasone $(400 \mu \mathrm{g}$ in $0.1 \mathrm{ml}$ ) was used in 83 of 94 cases $(88.3 \%)$. Systemic antibiotics were used only in 51 cases $(50.5 \%)$, mostly oral ciprofloxacin.

At final follow-up, 31 patients (30.7\%) had at least one vitrectomy. Data on final visual outcome was not available in 15 cases (14.9\%), due to loss to follow-up. Of 86 remaining cases, visual outcome was $6 / 36$ or better in only 20 cases $(23.3 \%)$. The majority of patients had poor visual outcomes. There were 17 patients $(19.8 \%)$ who were NLP, $12(14.0 \%)$ had an evisceration and nine (10.5\%) had an enucleation.

Final visual outcomes were stratified as either good (defined as a VA of 6/36 or better) or poor (VA of $6 / 60$ or worse), following categories used in the Endophthalmitis Vitrectomy Study (EVS). ${ }^{5}$ On univariate analysis, Table 2, patients with a presenting VA of LP or worse were more likely to have poor visual outcomes than those presenting with $\mathrm{HM}$ or better $(90.2 \%$ vs. $64.3 \%, \mathrm{p}=0.008)$. Patients with no view of the fundus at presentation were also more likely to end up with a VA of $6 / 60$ or worse $(82.7 \%$ vs. $30.0 \%, p=0.001)$. The presence of hypopyon and a history of diabetes were not significant predictors of poor visual outcome. 
On gram stain, a larger number of organisms seen (3+ vs. $2+$ vs. $0-1+)$ was associated with poor final VA ( $92.9 \%$ vs. $73.3 \%$ vs. $51.9 \%, p<0.001)$. This factor remained significant on multivariate analysis $(p=0.032)$, Table 3 . In addition, when Streptococci were readily identified on gram stain, cases had worse visual outcomes ( $89.7 \%$ vs. $65.2 \%, p=0.010)$. On culture, heavy microbial growth was more likely to be associated with poor visual outcome than moderate or light/no growth (93.3\% vs. $75.0 \%$ vs. $39.1 \%, p<0.001)$. However, the species of Streptococcus involved and the presence of a second microorganism did not correlate with final VA.

\begin{tabular}{|c|c|}
\hline Snellen visual acuity & Number of cases $(\mathbf{n = 1 0 1})$ \\
$6 / 9$ & 1 \\
$6 / 18$ & 2 \\
$6 / 24$ & 1 \\
$6 / 36$ & 2 \\
$6 / 60$ & 1 \\
Counting Fingers (CF) & 3 \\
Hand Movements (HM) & 36 \\
Light Perception (LP) & 43 \\
No Light Perception (NLP) & 8 \\
\hline
\end{tabular}

† Data not available for 4 cases

Table 1: Number of cases by presenting visual acuity

\begin{tabular}{|c|c|c|c|c|}
\hline Characteristic & $\mathbf{N}$ & $\begin{array}{l}\text { VA } 6 / 36 \text { or } \\
\text { better }(\%)\end{array}$ & $\begin{array}{l}\text { VA } 6 / 60 \text { or } \\
\text { worse (\%) }\end{array}$ & p value \\
\hline $\begin{array}{l}\text { Presenting visual acuity } \\
\text { LP or worse } \\
\text { HM or better }\end{array}$ & $\begin{array}{l}41 \\
42\end{array}$ & $\begin{array}{c}4(9.8) \\
15(35.7)\end{array}$ & $\begin{array}{l}37(90.2) \\
27(64.3)\end{array}$ & 0.008 \\
\hline $\begin{array}{l}\text { Fundus visible at } \\
\text { presentation }\end{array}$ & 10 & $7(70.0)$ & $3(30.0)$ & 0.001 \\
\hline
\end{tabular}




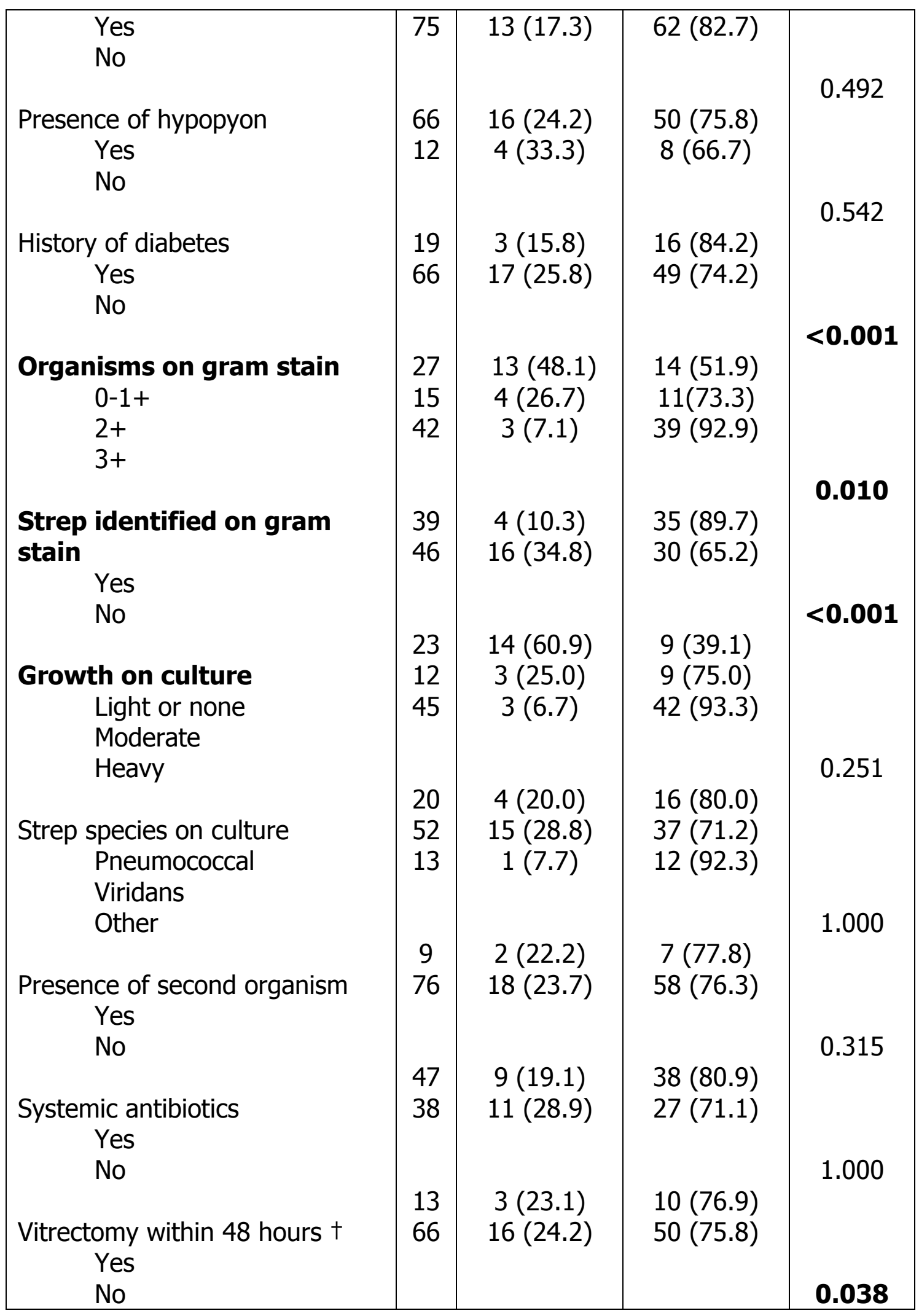




\begin{tabular}{|l|c|c|c|c|}
\hline No. of intravitreal injections & 24 & $10(41.7)$ & $14(58.3)$ & \\
& 35 & $7(20.0)$ & $28(80.0)$ & \\
1 & 20 & $2(10.0)$ & $18(90.0)$ & \\
2 & & & & \multirow{2}{*}{1.000} \\
3 or more & 71 & $17(23.9)$ & $54(76.1)$ & \\
$\quad$ & 8 & $2(25.0)$ & $6(75.0)$ & \\
$\begin{array}{c}\text { Intravitreal dexamethasone }+ \\
\text { Yes } \\
\text { No }\end{array}$ & & & & \\
\hline
\end{tabular}

† Excludes patients who proceeded directly to enucleation or evisceration N.B where $n \neq 86$, data was not available in patient records

Table 2: Characteristics associated with final visual outcome

\begin{tabular}{|l|c|c|}
\hline Characteristic & $\mathbf{p}$ & Odds ratio (95\% \\
value & CI) \\
\hline Presenting visual acuity of LP or worse & 0.367 & $2.056(0.429-9.859)$ \\
Fundus not visible at presentation & $\mathbf{0 . 0 4 4}$ & $\mathbf{8 . 9 5 8 ( 1 . 0 5 6 -}$ \\
History of diabetes & 0.241 & $\mathbf{7 5 . 9 6 2 )}$ \\
Large (3+) number of organisms on & $\mathbf{0 . 0 2 1}$ & $3.198(0.458-$ \\
gram stain ₹ & 0.923 & $22.311)$ \\
Streptococci identified on gram stain & 0.403 & $\mathbf{9 . 0 7 2 ( 1 . 4 0 3 -}$ \\
Non-viridans Streptococcal species on culture & 0.584 & $\mathbf{5 8 . 6 3 8 )}$ \\
Presence of second organism & 0.867 & $1.084(0.211-5.574)$ \\
Use of systemic antibiotics & 0.846 & $2.183(0.350-$ \\
Vitrectomy within 48 hours & 0.992 & $13.616)$ \\
Use of intravitreal dexamethasone & & $0.550(0.065-4.667)$ \\
& & $1.144(0.239-5.470)$ \\
& & $0.803(0.088-7.368)$ \\
& & $1.012(0.102-$ \\
& & $10.041)$ \\
\hline
\end{tabular}

₹ Adjusted for presenting visual acuity, visibility of fundus, history of diabetes, number of organisms on gram stain, type of organism identified, presence of second organism on culture, use of systemic antibiotics, vitrectomy within 48 hours, and use of intravitreal dexamethasone. 
\# Grading for amount of single organism seen on high-power oil immersion field (x1000) as described in methods.

N.B. Streptococcal groups divided into two - viridans vs. non-viridans Streptococci

Table 3: Multivariate analysis of predictors of poor visual outcome

The decision to perform early vitrectomy, within 48 hours of presentation, had no correlation with visual outcome ( $76.9 \%$ vs. $75.8 \%, p=1.000)$. The use of systemic antibiotic therapy and intravitreal dexamethasone also did not significantly affect the visual outcome. The need for a higher number of intravitreal injections performed was significantly associated with a poor final VA $(p=0.038)$.

For the secondary endpoint, patients were separated into groups who had removal of the eye (enucleation or evisceration, 21 patients) and no removal of the eye (65 patients). As illustrated in Table 4, patients with a presenting VA of LP or worse were more likely to lose the affected eye than patients with vision of $\mathrm{HM}$ or better ( $34.1 \%$ vs. $14.3 \%, p=0.042)$. In addition, patients who were infected with viridans group Streptococci were less likely to undergo enucleation or evisceration (11.5\% vs. $45.0 \%$ and $46.2 \%, p=0.002)$, and this association remained significant on multivariate analysis $(p=0.005)$, Table 5.

\begin{tabular}{|c|c|c|c|c|}
\hline Characteristic & $\mathbf{N}$ & $\begin{array}{c}\text { No } \\
\text { removal of } \\
\text { eye (\%) }\end{array}$ & $\begin{array}{c}\text { Removal } \\
\text { of eye (\%) }\end{array}$ & p value \\
\hline $\begin{array}{c}\text { Presenting visual acuity } \\
\text { LP or worse }\end{array}$ & 41 & $27(65.9)$ & $14(34.1)$ & $\mathbf{0 . 0 4 2}$ \\
HM or better & 42 & $36(85.7)$ & $6(14.3)$ & \\
$\begin{array}{c}\text { Fundus visible at presentation } \\
\text { Yes }\end{array}$ & 10 & $9(90.0)$ & $1(10.0)$ & 0.439 \\
No & 75 & $55(73.3)$ & $20(26.7)$ & \\
\hline
\end{tabular}




\begin{tabular}{|c|c|c|c|c|}
\hline Presence of hypopyon & & & & 0.152 \\
\hline Yes & 66 & $52(78.8)$ & $14(21.2)$ & \\
\hline No & 12 & $7(58.3)$ & $5(41.7)$ & \\
\hline History of diabetes & & & & 0.379 \\
\hline Yes & 19 & $16(84.2)$ & $3(15.8)$ & \\
\hline No & 66 & $48(72.7)$ & $18(27.3)$ & \\
\hline Organisms on gram stain & & & & 0.967 \\
\hline $0-1+$ & 27 & $20(74.1)$ & $7(25.9)$ & \\
\hline $2+$ & 15 & $11(73.3)$ & $4(26.7)$ & \\
\hline $3+$ & 42 & $32(76.2)$ & $10(23.8)$ & \\
\hline Strep identified on gram stain & & & & 0.458 \\
\hline Yes & 39 & $31(79.5)$ & $8(20.5)$ & \\
\hline No & 46 & $33(71.7)$ & $13(28.3)$ & \\
\hline Growth on culture & & & & 0.064 \\
\hline Light or none & 23 & $21(91.3)$ & $2(8.7)$ & \\
\hline Moderate & 12 & $10(83.3)$ & $2(16.7)$ & \\
\hline Heavy & 45 & $30(66.7)$ & $15(33.3)$ & \\
\hline Strep species on culture & & & & 0.002 \\
\hline Pneumococcal & 20 & $11(55.0)$ & $9(45.0)$ & \\
\hline Viridans & 52 & $46(88.5)$ & $6(11.5)$ & \\
\hline Other & 13 & $7(53.8)$ & $6(46.2)$ & \\
\hline Presence of second organism & & & & 0.441 \\
\hline Yes & 9 & $8(88.9)$ & $1(11.1)$ & \\
\hline No & 76 & $56(73.7)$ & $20(26.3)$ & \\
\hline Systemic antibiotics & & & & 0.129 \\
\hline Yes & 47 & $32(68.1)$ & $15(31.9)$ & \\
\hline No & 38 & $32(84.2)$ & $6(15.8)$ & \\
\hline Vitrectomy within 48 hours $\S$ & & & & 1.000 \\
\hline Yes & 13 & $11(84.6)$ & $2(15.4)$ & \\
\hline No & 66 & $52(78.8)$ & $14(21.2)$ & \\
\hline $\begin{array}{c}\text { No. of intravitreal injections } \S \\
1\end{array}$ & 24 & $18(75.0)$ & $6(25.0)$ & 0.050 \\
\hline
\end{tabular}




\begin{tabular}{|l|c|c|c|c|}
\hline 2 & 35 & $32(91.4)$ & $3(8.6)$ & \\
3 or more & 20 & $13(65.0)$ & $7(35.0)$ & \\
\begin{tabular}{|l} 
Intravitreal dexamethasone $\S$ \\
Yes
\end{tabular} & & & & \multirow{2}{*}{0.661} \\
No & 81 & $57(80.3)$ & $14(19.7)$ & \\
& 8 & $6(75.0)$ & $2(25.0)$ & \\
\hline
\end{tabular}

$\S$ Excludes patients who proceeded directly to enucleation or evisceration

N.B where $n \neq 86$, data was not available in patient records

Table 4: Characteristics associated with removal of the eye

\begin{tabular}{|l|c|c|}
\hline Characteristic & $\begin{array}{c}\mathbf{p} \\
\text { value }\end{array}$ & $\begin{array}{c}\text { Odds ratio (95\% } \\
\text { CI) }\end{array}$ \\
\hline Presenting visual acuity of LP or worse & 0.124 & $3.534(0.708-17.644)$ \\
Fundus not visible at presentation & 0.348 & $4.492(0.194-$ \\
History of diabetes & 0.217 & $103.864)$ \\
Large (3+) number of organisms on gram & 0.887 & $0.291(0.041-2.063)$ \\
stain ₹ & 0.336 & $0.865(0.117-6.379)$ \\
Streptococci identified on gram stain & $\mathbf{0 . 0 0 1}$ & $0.345(0.040-3.009)$ \\
Non-viridans Streptococcal species on & 0.999 & $\mathbf{2 7 . 5 4 7 ( 3 . 7 2 1 -}$ \\
culture & 0.903 & $\mathbf{2 0 3 . 9 2 5 )}$ \\
Presence of second organism & 0.647 & $0.000(\mathrm{~N} / \mathrm{A})$ \\
Use of systemic antibiotics & 0.765 & $1.106(0.220-5.568)$ \\
Vitrectomy within 48 hours & & $1.801(0.145-22.344)$ \\
Use of intravitreal dexamethasone & & $0.692(0.062-7.764)$ \\
\hline
\end{tabular}

I Adjusted for presenting visual acuity, visibility of fundus, history of diabetes, number of organisms on gram stain, type of organism identified, presence of second organism on culture, use of systemic antibiotics, vitrectomy within 48 hours, and use of intravitreal dexamethasone \# Grading for amount of single organism seen on high-power oil immersion field (x1000) as described in methods.

N.B. Streptococcal groups divided into two - viridans vs. non-viridans Streptococci

Table 5: Multivariate analysis of predictors of removal of the eye 
Early vitrectomy did not increase the likelihood of retaining the eye: $84.6 \%$ of patients with early vitrectomy retained their eye, compared to $78.8 \%$ of patients who initially received intravitreal injections only $(p=1.000)$. The use of systemic antibiotics or intravitreal dexamethasone was not associated with removal of the eye.

\section{DISCUSSION}

Patients with culture-positive Streptococci endophthalmitis and a presenting VA of LP or worse, or with no view of the fundus at presentation, are more likely to have a poor visual outcome and/or more likely to lose their eye, regardless of their aetiology. This finding agrees with the outcomes in previous studies including the EVS. ${ }^{5}$ Poor VA and poor view of fundus are surrogate markers for worse intraocular inflammation. They may also indicate underlying complications of endophthalmitis, such as retinal or choroidal detachment, which may lead to poorer outcomes. ${ }^{14}$

In this population of culture-positive Streptococci, most were readily visible on gram stain as gram-positive cocci, and were identifiable as resembling Streptococci in nearly half of cases. This study showed that early identification of Streptococcion gram stain significantly predicted poorer visual outcome. Our reporting laboratory finalises gram stain results within 24 hours, and results are often available within 6 hours. Therefore, early identification of Streptococcal endophthalmitis, known for its virulence, may allow early prognostication of outcome and more aggressive management. Although not previously reported in endophthalmitis, the utility of gram stain identification of Streptococci has been extensively investigated in community-acquired pneumonia and pharyngitis. ${ }^{15,16}$ Most studies show close 
concordance between gram stain and eventual culture results, suggesting that gram stain results can be used for early diagnosis and tailoring of suitable therapy.

In this cohort, a large number of organisms on microscopy and heavy growth on culture were associated with worse visual outcomes. Streptococci are fast growing organisms, and the vitreous is a nutritive and immune-privileged environment that can allow Streptococci to multiply quickly. Large numbers of organisms suggest a more virulent Streptococca/ infection leading to worse outcomes. ${ }^{17}$

The spectrum of Streptococcalspecies is similar to the prevalence of various species in the normal respiratory flora of healthy subjects. ${ }^{18,19}$ This observation supports the finding that endophthalmitis are often caused by nasopharyngeal commensals, particularly a patient's own nasal flora. ${ }^{20,21}$ Viridans group Streptococci, in particular, are rarely found in normal conjunctival flora, and some authors suggest that contamination may occur through aerosolisation when the patient, surgeon or staff are talking. ${ }^{22} 22$ The species of Streptococcus did not significantly influence the visual outcome, although patients who had viridans Streptococci fared best compared to others. This finding mirrors previous studies, where $S$. pneumoniae or $\beta$-haemolytic Streptococci were associated with worse visual outcomes, compared to viridans group Streptococci ${ }^{6,7}$ Although the trend for VA is not significant, patients with viridans Streptococciendophthalmitis were significantly less likely to lose their eye. Viridans Streptococci are relatively lower in virulence compared to other Streptococci, due to lack of virulence factors such as streptolysins and exotoxins, and may therefore be less damaging to ocular tissues. ${ }^{23}$

As expected, the final visual outcome is poor: $44.2 \%$ have no remaining vision, a percentage that is comparable to existing literature on Streptococcal endophthalmitis (28.6-58.3\%). ${ }^{6-8,10}$ Nearly one-quarter of patients undergo enucleation or 
evisceration, emphasising the poor outcome of this patient cohort; in contrast, only $1 \%$ of patients in the EVS study lose their infected eye. ${ }^{5}$ In this cohort, only $15.3 \%$ recovered vision of at least $6 / 18$, slightly better than reported in previous studies $(8.3-14.3 \%))^{6-8,} 10$

In our institution, pars plana vitrectomy is performed if there is lack of improvement or clinical worsening at 24-48 hours, despite intravitreal antibiotics. Our data shows that early vitrectomy within 48 hours does not improve visual outcome. Vitrectomy aims to remove inflammatory debris and prevent further damage, and although few reports suggest improvement in outcomes in some endophthalmitis, ${ }^{11}$ this study does not show any benefit in Streptococcalendophthalmitis. One other study on Streptococcalendophthalmitis reported a non-significant trend of worse outcomes in patients who underwent vitrectomy, compared to intravitreal tap and injection alone $(80 \%$ vs. $60 \%, p=0.402) .{ }^{8}$ There are several possible reasons for these findings. Firstly, Streptococci are virulent bacteria capable of producing severe inflammation, and damage to ocular tissues may occur early in the course of infection, leaving minimal chance for any improvement even with early vitrectomy. Experimental studies of gram-positive endophthalmitis showed that intraocular inflammation was observed as early as 3 hours post-inoculation, with maximal inflammation from 12 hours onwards. ${ }^{24}$ Early vitrectomy at our institution was within a practical 48 hours, while the EVS protocol mandated that all vitrectomies were performed within six hours. Secondly, vitrectomy in inflamed eyes is riskier due to poorer visibility and friable tissues, increasing the likelihood of iatrogenic complications such as retinal detachment. ${ }^{11}$ Thirdly, this study is observational and non-randomised. Patients were chosen for vitrectomy at the treating surgeon's discretion. Therefore, it is possible that patients were chosen for vitrectomy because of worse clinical presentation or lack of response to intravitreal therapy. If patients in the early 
vitrectomy group had worse disease and worse outcomes, any benefit of vitrectomy may be masked.

This study has a number of strengths. Data were collected over fifteen years at a single centre, and there was minimal variability in data collection, treatment protocols and equipment. Samples were sent to a single microbiology laboratory, allowing standardisation of testing and reporting. A minimum of six months of follow-up was imposed for final visual acuity data. Several limitations should also be noted. Due to the observational nature of the study, the possibility of selection bias could not be excluded. There are also limitations arising from incomplete data, particularly missing data points for final visual acuity, as some patients were lost to follow-up (15\%). In addition, due to the small sample size, statistical significance could not be determined from subgroup analyses, particularly based on aetiology. Further to that, an observed finding in our study was that some factors, upon univariate analysis (Table 2), were statistically significant however upon multivariate analysis (Table 4) were no longer statistically significant. This implicates confounders contributing to several of the findings seen in Table 2 and should be considered in the interpretation of the results. Another consideration is that with our retrospective study design, it is difficult to interpret the relationship between treatment options and outcomes. This is because in practicality, disease severity (determined through patient symptoms and examination findings) influences treatment decisions with respect to need for vitrectomy, number of injections, use of steroids and antibiotics, confounding our interpretation. Lastly, vitrectomy technology evolved considerably over the duration of the study, with later years incorporating technologies and techniques to improve safety. These include moving from 20-gauge to 25-gauge valved pars plana ports, the use of wide-field non-contact viewing systems and utilisation of smaller aperture ocutomes with faster cut rates and valved cannulae. Unfortunately the numbers in our study are too small to perform meaningful analysis 
into the significance of these improvements in vitrectomy technique. Despite this, improvements in outcomes are expected and expert opinion has noted improvements in the safety of vitrectomy with the technological advancements leading to less iatrogenic retinal breaks, an important complication to avoid in the setting of endophthalmitis due to the high risk of development of proliferative vitreoretinopathy (PVR). ${ }^{25,26}$ Continued data collection may further clarify the role of modern vitrectomy in virulent endophthalmitis.

Our findings confirm that, disappointingly, Streptococcal endophthalmitis has devastating sequelae in our cohort, with florid inflammation leading to poor visual outcomes. The prime focus for treating clinicians should be early detection and early treatment to minimize disease impact. Patient education and regular re-education following intraocular procedures is a vital process. Following dissemination of our findings in our department, consideration of early vitrectomy in the management of endophthalmitis is being sought. With recent advances in vitrectomy techniques, we feel that for research clinicians there can only be one recommendation, that a multicentred, multi-regional prospective randomized clinical control trial that includes an arm for immediate vitrectomy (within 4-6 hours of presentation). This protocol should include close collaboration with microbiologists.

Clinical presentation and microbiological results can provide useful information on visual outcome in Streptococca/endophthalmitis. In the right setting, microscopy results can be available within hours of specimen collection, and culture results shortly after. In fact, polymerase chain reaction (PCR) testing of Streptococci now allows even faster identification of pathogens using known genes like pneumolysin and autolysin. ${ }^{27}$ Given the ability to identify this devastating pathogen early, clinicians can make earlier treatment decisions to attempt to improve visual 
outcomes. Rapid diagnosis also better prepares the treating physician to counsel patients on their prognosis.

\section{Acknowledgements}

The authors would like to thank all staff involved in this study.

Contributors: All authors (EDK, JRR, SSS, PJA) contributed to design, collection, analysis and interpretation of data, and review of manuscript. 


\section{REFERENCES}

1. Leng T, Miller D, Flynn HW, Jr., Jacobs DJ, Gedde SJ. Delayed-onset blebassociated endophthalmitis (1996-2008): causative organisms and visual acuity outcomes. Retina 2011;31 2: 344-52.

2. McCannel CA. Meta-analysis of endophthalmitis after intravitreal injection of Anti-vascular Endothelial Growth Factor agents: Causative Organisms and Possible Prevention Strategies. Retina 2011;31 4: 654-61.

3. Keynan Y, Finkelman Y, Lagace-Wiens P. The microbiology of endophthalmitis: global trends and a local perspective. European Journal of Clinical Microbiology \& Infectious Diseases 2012;31 11: 2879-86.

4. Lalwani GA, Flynn HW, Scott IU, Quinn CM, Berrocal AM, Davis JL, et al. Acute-onset endophthalmitis after clear corneal cataract surgery (1996-2005). Ophthalmology 2008;115 3: 473-76.

5. Endophthalmitis Vitrectomy Study Group. RESULTS OF THE ENDOPHTHALMITIS VITRECTOMY STUDY - A RANDOMIZED TRIAL OF IMMEDIATE VITRECTOMY AND OF INTRAVENOUS ANTIBIOTICS FOR THE TREATMENT OF POSTOPERATIVE BACTERIAL ENDOPHTHALMITIS. Arch Ophthalmol 1995;113 12: 1479-96.

6. Jones S, Cohen EJ, Arentsen JJ, Laibson PR. Ocular streptococcal infections. Cornea 1988;7 4: 295-9.

7. Mao LK, Flynn HW, Jr., Miller D, Pflugfelder SC. Endophthalmitis caused by streptococcal species. Arch Ophthalmol 1992;110 6: 798-801.

8. Miller JJ, Scott IU, Flynn HW, Jr., Smiddy WE, Corey RP, Miller D. Endophthalmitis caused by Streptococcus pneumoniae. Am J Ophthalmol 2004;138 2: 231-6.

9. Soriano F, Perez-Trallero E, Pallares R, Meseguer MA, Fleites A, Gene A, et al. Streptococcus pneumoniae endophthalmitis: a study of 36 cases with special 
reference to antibiotic resistance and treatment options. Clin Microbiol Infect 2006;12 6: 519-26.

10. Goldberg RA, Flynn HW, Jr., Miller D, Gonzalez S, Isom RF. Streptococcus Endophthalmitis Outbreak after Intravitreal Injection of Bevacizumab: One-Year Outcomes and Investigative Results. Ophthalmology 2013.

11. Kuhn F, Gini G. Ten years after... are findings of the Endophthalmitis Vitrectomy Study still relevant today? Graefes Arch Clin Exp Ophthalmo/ 2005;243 12: $1197-9$.

12. Shah GK, Stein JD, Sharma S, Sivalingam A, Benson WE, Regillo CD, et al. Visual outcomes following the use of intravitreal steroids in the treatment of postoperative endophthalmitis. Ophthalmology 2000;107 3: 486-9.

13. Hall EF, Scott GR, Musch DC, Zacks DN. Adjunctive intravitreal dexamethasone in the treatment of acute endophthalmitis following cataract surgery. Clin Ophthalmo/2008;2 1: 139-45.

14. Dacey MP, Valencia M, Lee MB, et al. Echographic findings in infectious endophthalmitis. Arch Ophthalmol 1994;112 10: 1325-33.

15. Bohte R, Hermans J, vandenBroek PJ. Early recognition of Streptococcus pneumoniae in patients with community-acquired pneumonia. European Journal of Clinical Microbiology \& Infectious Diseases 1996;15 3: 201-05.

16. Roson B, Carratala J, Verdaguer R, Dorca J, Manresa F, Gudiol F. Prospective study of the usefulness of sputum gram stain in the initial approach to communityacquired pneumonia requiring hospitalization. Clinical Infectious Diseases 2000;31 4: 869-74.

17. Ryan S, Schachat A, Wilkinson C, Hinton D, Sadda S, Wiedemann P. Retina, 5th ed. Chapter 122: Infectious Endophthalmitis. Saunders; 2012. p. 2019-39. 18. Konno M, Baba S, Mikawa H, Hara K, Matsumoto F, Kaga K, et al. Study of upper respiratory tract bacterial flora: first report. Variations in upper respiratory tract bacterial flora in patients with acute upper respiratory tract infection and 
healthy subjects and variations by subject age. J Infect Chemother 2006;12 2: 8396.

19. Hable KA, Washington JA, Herrmann EC. Bacterial and Viral Throat Flora: Comparison of Findings in Children with Acute Upper Respiratory Tract Disease and in Healthy Controls during Winter. Clinical Pediatrics 1971;10 4: 199-203.

20. Bannerman TL, Rhoden DL, McAllister SK, Miller JM, Wilson LA. The source of coagulase-negative staphylococci in the Endophthalmitis Vitrectomy Study. A comparison of eyelid and intraocular isolates using pulsed-field gel electrophoresis. Arch Ophthalmol 1997;115 3: 357-61.

21. Speaker MG, Milch FA, Shah MK, Eisner W, Kreiswirth BN. Role of external bacterial flora in the pathogenesis of acute postoperative endophthalmitis. Ophthalmology 1991;98 5: 639-49; discussion 50.

22. Chen E, Lin MY, Cox J, Brown DM. ENDOPHTHALMITIS AFTER INTRAVITREAL INJECTION The Importance of Viridans Streptococci. Retina-the Journal of Retinal and Vitreous Diseases 2011;31 8: 1525-33.

23. Brooks G, Carroll K, Butel J, Morse S, Mietzner T. Medical Microbiology. 26th ed: McGraw-Hill Medical; 2012.

24. Callegan MC, Booth MC, Jett BD, Gilmore MS. Pathogenesis of gram-positive bacterial endophthalmitis. Infect Immun 1999;67 7: 3348-56.

25. Pastor JC, de la Rúa ERg, Martín F. Proliferative vitreoretinopathy: risk factors and pathobiology. Progress in retinal and eye research 2002;21 1: 127-44.

26. Sadaka A, Giuliari GP. Proliferative vitreoretinopathy: current and emerging treatments. Clinical ophthalmology (Auckland, NZ) 2012;6: 1325.

27. Suzuki N, Yuyama M, Maeda S, Ogawa H, Mashiko K, Kiyoura Y. Genotypic identification of presumptive Streptococcus pneumoniae by PCR using four genes highly specific for S. pneumoniae. Journal of Medical Microbiology 2006;55 6: 70914. 


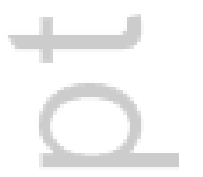




\section{University Library}

\section{- M M N E R VA A gateway to Melbourne's research publications}

Minerva Access is the Institutional Repository of The University of Melbourne

Author/s:

Kurniawan, ED;Rocke, JR;Sandhu, SS;Allen, PJ

Title:

Predictors of visual outcome and the role of early vitrectomy in streptococcal endophthalmitis

Date:

2018-05-01

\section{Citation:}

Kurniawan, E. D., Rocke, J. R., Sandhu, S. S. \& Allen, P. J. (2018). Predictors of visual outcome and the role of early vitrectomy in streptococcal endophthalmitis. CLINICAL AND EXPERIMENTAL OPHTHALMOLOGY, 46 (4), pp.424-431. https://doi.org/10.1111/ceo.13077.

Persistent Link:

http://hdl.handle.net/11343/293813 